
АНТИРЕТРОВИРУСНУЮ ТЕРАПИЮ

( Т.Б. Моргунова* , А.А. Зорина, Е.С. Малолеткина, Ю.П. Сыч, А.В. Васильева, В.В. Фадеев

Первый Московский государственный медицинский университет им. И.М. Сеченова (Сеченовский Университет), Москва, Россия

Статья посвящена клиническому случаю развития болезни Грейвса у пациентки с ВИЧ-инфекцией, получающей антиретровирусную терапию. За последние десятилетия число ВИЧ-инфицированных пациентов во всем мире существенно возросло. Применяемая на сегодняшний день высокоактивная антиретровирусная терапия позволяет существенно улучшить прогноз для этих пациентов. Однако ее применение сопряжено с рядом осложнений, в частности, развитием синдрома восстановления иммунитета, на фоне чего возможно развитие таких аутоиммунных заболеваний, как болезнь Грейвса, полимиозит и синдром Гийена-Барре. Поэтому хотелось бы обратить внимание врачей на возможность развития такого осложнения у больных, получающих антиретровирусную терапию. Своевременная диагностика и лечение нарушений функции щитовидной железы позволят избежать осложнений, сопряженных с избытком или дефицитом тиреоидных гормонов.

КЛЮЧЕВЫЕ СЛОВА: кЛИнический случай, болезнь Грейвса, ВИЧ-инфекция, высокоактивная антиретровирусная терапия, синдром восстановления иммунитета.

\title{
GRAVES' DISEASE IN HIV PATIENT RECEIVING ANTIRETROVIRAL THERAPY
}

(c) Tatyana B. Morgunova*, Anastasia A. Zorina, Ekaterina S. Maloletkina, Yulia P. Sytch, Ariadna V. Vasileva, Valentin V. Fadeyev

I.M. Sechenov First Moscow State Medical University (Sechenov University), Moscow, Russia

The article focuses on the clinical case of Graves' disease in a patient with HIV infection who is receiving antiretroviral therapy. The number of HIV-infected patients has increased significantly in recent decades all over the world. The currently used highly active antiretroviral therapy can significantly improve the prognosis for these patients. However, its use is associated with a number of complications, in particular the development of immune reconstitution syndrome, under which the development of such autoimmune diseases as Graves' disease, polymyositis and Guillain-Barre syndrome may occur. Therefore, we would like to draw the attention of doctors to the possibility of such a complication in patients receiving antiretroviral therapy. Timely diagnosis and treatment of thyroid disorders will help to avoid the complications associated with an excess or deficit of thyroid hormones.

KEYWORDS: case report, Graves' disease, HIV infection, highly active antiretroviral therapy, immune reconstitution syndrome.

\section{АКТУАЛЬНОСТЬ}

В последние десятилетия для лечения пациентов с ВИЧ-инфекцией в клиническую практику стали активно внедрять различные комбинации антиретровирусных препаратов. Проводимая на сегодняшний день высокоактивная антиретровирусная терапия (ВААРТ) позволила, с одной стороны, в определенной мере контролировать течение ВИЧ-инфекций, что привело к уменьшению показателей заболеваемости и смертности у ВИЧ-инфицированных пациентов. С другой стороны, был отмечен ряд нежелательных явлений, ассоциированных с данной терапией. Одним из них является синдром восстановления иммунитета. В литературе можно встретить и другие названия данного синдрома: синдром иммунной перестройки, синдром реконструкции иммунной системы, воспалительный синдром иммунной перестройки и другие. К наиболее частым неинфекционным проявлениям данного синдрома относятся болезнь Грейвса, саркоидоз, системная красная волчанка, полимиозит и другие заболевания. В данной статье мы хотим представить клиническое наблюдение пациентки с ВИЧ-инфекцией и болезнью Грейвса, развившейся на фоне проводимой антиретровирусной терапии.

\section{ОПИСАНИЕ СЛУЧАЯ}

Пациентка А., 38 лет, в апреле 2019 г. обратилась в Клинику эндокринологии Университетской клинической больницы № 2 ФГАОУ ВО «Первый МГМУ им. И.М. Сеченова» Минздрава России (Сеченовский Университет) с жалобами на слабость, головокружение, потливость, эмоциональную лабильность, слезотечение.

Из анамнеза. В 2004 г. у пациентки выявлена ВИЧ-инфекция. С 2016 г. получает антиретровирусную терапию по схеме: лопинавир/ритонавир 200 мг + 50 мг 3 таблетки в стуки, ламивудин 150 мг 1 таблетка в сутки. Иммунный статус от 02.2019: CD4 683 (35\%), CD8 706 (36\%), CD4/CD8 0,97, ПЦР РНК ВИЧ от 02.2019 - не обнаружена.

Впервые жалобы на головокружение, дрожь в теле, раздражительность, потливость, «перебои» в работе сердца появились в июне 2018 г. Также с этого времени пациентка отметила появление экзофтальма, стали беспокоить жалобы на чувство давления за глазами, 
Таблица 1. Динамика уровня гормонов и антител к рецептору ТТГ

\begin{tabular}{|c|c|c|c|c|}
\hline Дата исследования & $\begin{array}{c}\text { ТТГ } \\
(0,4-4,0 \text { мЕд/л) }\end{array}$ & свободный Т4 & свободный Т3 & $\begin{array}{c}\text { AT-рТТГ } \\
\text { (0-1 ME/ת) }\end{array}$ \\
\hline 07.06.2018 & $<0,01$ & $\begin{array}{c}>7,7 \\
(0,61-1,12 \text { нг/дл) }\end{array}$ & - & - \\
\hline 02.08.2018 & 68,86 & $\begin{array}{c}0,04 \\
(0,61-1,12 \text { нг/дл) }\end{array}$ & - & - \\
\hline 21.11 .2018 & 1,5 & $\begin{array}{c}<0,3 \\
(0,89-1,76 \text { нг/дл) }\end{array}$ & $\begin{array}{c}2,25 \\
(1,8-4,2 \text { пг/мл) }\end{array}$ & - \\
\hline 15.04 .2019 & 0,27 & $\begin{array}{c}0,16 \\
(0,89-1,76 \text { нг/дл) }\end{array}$ & $\begin{array}{c}1,81 \\
(1,8-4,2 \text { пг/мл) }\end{array}$ & 39,34 \\
\hline 29.04.2019 & 15,1 & $\begin{array}{c}<3,9 \\
(11,5-23,2 \text { пмоль/л) }\end{array}$ & $\begin{array}{c}4,4 \\
(3,5-6,5 \text { пмоль/л) }\end{array}$ & \\
\hline
\end{tabular}

двоение при взгляде вверх, слезотечение, покраснение век. При обследовании выявлен манифестный тиреотоксикоз (табл. 1), по данным ультразвукового исследования объем щитовидной железы (ЩЖ) составил 48,1 см³. На основании жалоб и результатов обследования пациентке был поставлен диагноз «болезнь Грейвса: диффузный токсический зоб ІІ ст., эндокринная офтальмопатия». Назначена терапия тиреостатиками: тиамазол в дозе 30 мг в сутки с последующим снижением дозы препарата до 15 мг в сутки. На фоне проводимой тиреостатической терапии отмечено субъективное улучшение самочувствия: не беспокоили жалобы на дрожь в теле и учащенное сердцебиение, уменьшилось чувство давления за глазами, слезотечение, гиперемия век. С пациенткой обсуждался вопрос радикального лечения (радиойодтерапия), однако от данного варианта лечения был получен категорический отказ. В период с июня 2018 г. по апрель 2019 г. наблюдалась врачами разных медицинских учреждений. В ноябре 2018 г. года пациентка переведена на схему «блокируй-замещай»: тиамазол 20 мг в сутки с последующим уменьшением дозы до 10 мг в сутки, левотироксин 25 мкг. Динамика уровня тиреотропного гормона (ТТГ) и тиреоидных гормонов представлена в таблице 1. При контрольном ультразвуковом исследовании ЩЖ в ноябре 2018 г. объем железы составил 72,3 см³ клинических симптомов компрессионного синдрома отмечено не было.

\section{Результаты физикального, лабораторного}

\section{и инструментального исследования}

Данные физикального осмотра. Вес - 48 кг, рост $161 \mathrm{~cm}$, ИМТ $=18,52$ кг/м². Кожные покровы физиологической окраски, умеренной влажности, чистые. Подкожножировая клетчатка развита умеренно, распределена равномерно. Отеков нет. В легких дыхание везикулярное, хрипов нет. Частота дыхания (ЧД) - 17 в минуту. Тоны сердца приглушены, ритмичные, шумов нет. АД 100/75 мм рт. ст. Пульс — 82 в минуту, ритмичный. Живот округлой формы, мягкий, при пальпации безболезненный во всех отделах. Печень по передней подмышечной линии по краю реберной дуги. Симптом поколачивания отрицательный с обеих сторон. ЩЖ равномерно увеличена (II ст.), безболезненна при пальпации, мягко-эластической консистенции, узловые образования не пальпируются. Обращает на себя внимание экзофтальм OU.
Активность орбитопатии по шкале CAS - 1 балл OU. Симптом Грефе - положительный; симптомы Дальримпля, Кохера, Штельвага, Розенбаха - отрицательные.

Показатели общего и биохимического анализов крови - в пределах референсного диапазона. Результаты исследования ТТГ и тиреоидных гормонов представлены в таблице 1.

УЗИ ЩЖ (06.05.19): ЩЖ расположена типично. Размеры: перешеек - 5,0 мм, правая доля - длина 87 мм, толщина 37 мм, ширина 43 мм, левая доля - длина 90 мм, толщина 34 мм, ширина 39 мм. Объем ЩЖ: 123 см³. Контуры ровные, капсула не утолщена, уплотнена; эхогенность диффузно умеренно пониженная. Эхоструктура ткани однородная. В режиме цветового допплеровского картирования (ЦДК) сосудистый рисунок значительно диффузно усилен. Линейная скорость кровотока по задней щитовидной артерии 150 cm/c. Региональные лимфоузлы: патологического увеличения не выявлено.

\section{ИСХОД И РЕЗУЛЬТАТЫ ПОСЛЕДУЮЩЕГО НАБЛЮДЕНИЯ}

Принимая во внимание клиническую картину заболевания (в том числе наличие у пациентки эндокринной орбитопатии), результаты лабораторных исследований (повышенный уровень антител к рецептору ТТГ), диффузное увеличение ЩЖ, повышение васкуляризации железы по данным ультразвукового исследования, болезнь Грейвса сомнений не вызывает. На фоне проводимой тиреостатической терапии у пациентки развился ятрогенный гипотиреоз. Проведена коррекция терапии. Пациентка проинформирована обо всех возможных вариантах лечения болезни Грейвса (медикаментозное, радикальное). Принимая во внимание высокий уровень антител к рецептору ТТГ, большой объем щЖ, а также желание самой пациентки вариантом выбора является хирургическое лечение (тиреоидэктомия). В дальнейшем пациентке была выполнена тиреоидэктомия, назначена заместительная терапия левотироксином в дозе 100 мкг. Послеоперационный период протекал без особенностей, состояние пациентки оставалось стабильно удовлетворительным. По результатам гистологического исследования: морфологическая картина диффузного токсического зоба. В анализе крови от июня 2019 г. (через 2 месяца после оперативного лечения) уровень ТТГ 1,5 мкMЕ/мл (0,4-4,0). 


\section{ОБСУЖДЕНИЕ}

ВИЧ-инфекция - прогрессирующее антропонозное заболевание с преимущественно контактным механизмом заражения, характеризующееся специфическим поражением иммунной системы с развитием иммунодефицита, который проявляется развитием оппортунистических инфекций, злокачественных новообразований, возникновением аутоиммунных реакций. Внедрение в клиническую практику ВААРТ способствовало переходу ВИЧ-инфекции из категории смертельных болезней в хроническое заболевание с большой ожидаемой продолжительностью жизни.

Применение активной антиретровирусной терапии обеспечивает клинически значимое повышение количества клеток $\mathrm{CD}^{+}{ }^{+}$Т-клеток, что сопровождается уменьшением риска прогрессирования заболевания, а также снижением риска оппортунистических инфекций. Вместе с тем длительная ВААРТ приводит к развитию ряда аутоиммунных заболеваний, обусловленных синдромом восстановления иммунитета [1]. Патогенез развития данного синдрома связан с качественным и количественным изменением $\mathrm{CD}^{+}$лимфоцитов. В ранний период применения ВААРТ происходит увеличение количества данных клеток за счет перераспределения их в организме пациента. Они поступают в кровоток из периферических лимфоузлов, где в основном сосредоточены активированные лимфоциты. Это создает предпосылки для запуска вторичного иммунного ответа на уже имеющиеся у больного неинфекционные и инфекционные антигены. Значительное увеличение абсолютного количества CD4+ приводит к усилению антигенспецифического иммунного ответа и продукции медиаторов воспаления, что может привести к образованию аутоантител и инфильтрации тканей активированными лимфоцитами. Аутоиммунные тиреопатии у пациентов с ВИЧ, получающих ВААРТ, встречаются в 1,5-2 раза чаще, чем в общей популяции: примерно у 3,0\% у женщин и 0,2\% мужчин [2]. Наиболее часто развивается болезнь Грейвса (88\% случаев), гораздо реже тиреоидит Хашимото (6\%) и гипотиреоз без повышения антител к ткани щЖ (6\%). Аутоиммунное поражение ЩЖ может развиться спустя несколько месяцев, иногда несколько лет после начала антиретровирусной терапии [3, 4]. Так, по данным Rasul S. и соавт., у 4 пациентов болезнь Грейвса манифестировала через 19-53 месяца от начала антиретровирусной терапии [4]. В работе Hsu E. и соавт. показано, что у пяти пациентов болезнь Грейвса манифестировала через 23-96 месяцев [5]. Учитывая высокую распространенность нарушений функции ЩЖ, сопутствующих иммуновосстановительной терапии, Европейская тиреоидная ассоциация (ETA) предлагает следующий подход к ведению таких пациентов [6].

Перед началом терапии всем пациентам рекомендуется определять уровень ТТГ, при выявлении отклонений - уровни свободных тироксина (Т4) и трийодтиронина (Т3). Рутинное определение антител к тиреопероксидазе (АТ-ТПО), антител к рецептору ТТГ (АТрТТГ), а также визуализация щЖ не рекомендуются. Наличие у пациента нарушения функции щЖ в анамнезе либо на момент планируемой ВААРТ не является противопоказанием к проведению ВААРТ, однако требует предварительного лечения и последующего наблюде- ния у эндокринолога. Внимательно необходимо отнестись к пациентам с ремиссией болезни Грейвса после консервативного лечения, в случае радикального лечения ожидаемый риск рецидива низкий. Противопоказаниями к ВААРТ являются активная стадия эндокринной офтальмопатии и наличие заболевания сердца, при котором тиреотоксикоз может представлять угрозу для здоровья. ЕТА рекомендует лечение этих состояний до проведения ВААРТ [6].

После проведения ВААРТ, в отличие от других вариантов иммуновосстановительной терапии, не рекомендуется рутинное динамическое определение ТТГ. Оценка уровня ТТГ показана только при развитии клинических проявлений патологии ЩЖ: потливость, неожиданные колебания веса, нервозность, учащенное сердцебиение и нарастающая утомляемость; следует предупредить пациента о необходимости обратиться за медицинской помощью при появлении этих симптомов. Данная рекомендация ETA согласуется с рекомендациями по ведению пациентов с ВИЧ Американского общества инфекционных заболеваний, может быть объяснена меньшей распространенностью патологии ЩЖ при применении ВААРТ в сравнении с другой иммуновосстановительной терапией. В поперечном исследовании Silva G.A. и соавт. у 117 пациентов с ВИЧ-инфекцией распространенность нарушений функции ЩЖ составила 34\%, большинство имели субклинический или манифестный гипотиреоз, однако в 13\% случаев выявлялся низкий свободный Т4 при нормальном или сниженном ТТГ, у 1\% выявили изолированное повышение свободного Т4, что может свидетельствовать о патологии гипофиза либо синдроме эутиреоидной патологии [6, 7].

Рутинная визуализация ЩЖ данной группе пациентов также не показана. В отношении длительности наблюдения четких рекомендаций не сформулировано, описано развитие болезни Грейвса у пациентов с ВИЧ в течение 8-33 месяцев (в среднем 17 месяцев) после начала ВААРТ.

Особенностью клинического течения болезни Грейвса после ВААРТ является атипичный, волнообразный характер, лабораторные изменения могут не соответствовать клинической симптоматике. При обнаружении у пациента уровня ТТГ в пределах 0,10-0,39 мЕД/л необходимо повторное определение ТТГ в течение 1 месяца, при уровне ТТГ <0,1 мЕД/л рекомендуется оценка уровня ТТГ, свободных Т4 и Т3 через 2 недели. Также необходимо провести тщательный сбор анамнеза и физикальное обследование согласно симптомам. В течение 2-4 недель после двукратного лабораторного подтверждения нарушения функции ЩЖ пациент должен быть направлен к эндокринологу. Дальнейшая диагностика включает определение АТрТТГ, при их повышении устанавливается диагноз болезни Грейвса.

Методом выбора при болезни Грейвса у пациентов с ВИЧ после ВААРТ является консервативное лечение по схеме «блокируй и замещай» в течение 12-18 месяцев, с последующей отменой и контролем рецидива согласно общепринятому подходу. За исключением случаев симптомного течения и пациентов из группы высокого риска осложнений тиреотоксикоза, лечение антитиреоидными препаратами следует начинать после трехмесячного периода наблюдения. 
При непереносимости тиреостатиков, неконтролируемом волнообразном течении заболевания и по предпочтению пациента, а также в случае рецидива после консервативной терапии показано радикальное лечение в объеме тиреоидэктомии/радиойодтерапии [6].

У нашей пациентки на фоне проводимой 3-компонентной ВААРТ получен адекватный иммунный ответ, и через 24 месяца от начала лечения появились клинические проявления тиреотоксикоза, у нее была диагностирована болезнь Грейвса. В большинстве случаев симптомы тиреотоксикоза появляются в течение первых 12 месяцев лечения. Как правило, у пациентов нет других аутоиммунных заболеваний, часто не отягощен семейный анамнез по аутоиммунной патологии [5]. На сегодняшний день не выделено четких факторов риска развития болезни Грейвса у пациентов с ВИЧ-инфекцией на ВААРТ. В литературе обсуждается вопрос о возможной ассоциации болезни Грейвса с полиморфизмом генов МНC $[8,9]$. Очевидно, чаще болезнь Грейвса будет развиваться у пациентов, получающих также противовирусную терапию препаратами интерферона по поводу гепатита C.

\section{ЗАКЛЮЧЕНИЕ}

Учитывая рост заболеваемости ВИЧ-инфекцией, широкое применение ВААРТ, в литературе появляется все больше сообщений о развитии нарушений функции щЖ у таких пациентов. Поэтому хотелось бы обратить внимание врачей на эту проблему у больных, получающих комбинированную антиретровирусную терапию.

\section{ДОПОЛНИТЕЛЬНАЯ ИНФОРМАЦИЯ}

Источник финансирования. Рукопись подготовлена на личные средства авторов.

Согласие пациента. Пациент добровольно подписал информированное согласие на публикацию персональной медицинской информации в обезличенной форме.

Конфликт интересов. Авторы декларируют отсутствие явных и потенциальных конфликтов интересов, связанных с публикацией настоящей статьи.

Участие авторов. Все авторы внесли значимый вклад и подготовку статьи, прочли и одобрили финальную версию перед публикацией.

\title{
СПИСОК ЛИТЕРАТУРЫ | REFERENCES
}

1. Roszkiewicz J, Smolewska E. Kaleidoscope of autoimmune diseases in HIV infection. Rheumatol Int. 2016;36(11):1481-1491. doi: 10.1007/s00296-016-3555-7.

2. Chen F, Day SL, Metcalfe RA, et al. Characteristics of autoimmune thyroid disease occurring as a late complication of immune reconstitution in patients with advanced human immunodeficiency virus (HIV) disease. Medicine (Baltimore). 2005;84(2):98-106. doi: 10.1097/01.md.0000159082.45703.90.

3. Jubault V, Penfornis A, Schillo F, et al. Sequential occurrence of thyroid autoantibodies and Graves' disease after immune restoration in severely immunocompromised human immunodeficiency virus1-infected patients. J Clin Endocrinol Metab. 2000;85(11):4254-4257. doi: 10.1210/jcem.85.11.6988

4. Rasul S, Delapenha R, Farhat F, et al. Graves' disease as a manifestation of immune reconstitution in HIV-Infected individuals after initiation of highly active antiretroviral therapy. AIDS Res Treat. 2011;2011:743597. doi: 10.1155/2011/743597.
5. Hsu E, Phadke VK, Nguyen ML. Short communication: hyperthyroidism in human immunodeficiency virus patients on combined antiretroviral therapy: case series and literature review. AIDS Res Hum Retroviruses. 2016:32(6):564-566. doi: 10.1089/AID.2015.0374.

6. Muller I, Moran C, Lecumberri B, et al. 2019 European thyroid association guidelines on the management of thyroid dysfunction following immune reconstitution therapy. Eur Thyroid J. 2019;8(4):173-185. doi: 10.1159/000500881

7. Silva GA, Andrade MC, Sugui Dde A, et al. Association between antiretrovirals and thyroid diseases: a cross-sectional study. Arch Endocrinol Metab. 2015;59(2):116-122. doi: 10.1590/2359-3997000000023.

8. Weetman AP. Thyroid abnormalities. Endocrinol Metab Clin North Am. 2014;43(3):781-790. doi: 10.1016/j.ecl.2014.05.006.

9. French MA, Lewin SR, Dykstra C, et al. Graves' disease during immune reconstitution after highly active antiretroviral therapy for HIV infection: evidence of thymic dysfunction. AIDS Res Hum Retroviruses. 2004;20(2):157-162. doi: 10.1089/088922204773004879.

Рукопись получена: 06.04.2020. Одобрена к публикации: 16.07.2020. Опубликована online: 03.08.2020.

\section{ИНФОРМАЦИЯ ОБ АВТОРАХ [AUTHORS INFO]}

\begin{abstract}
* Моргунова Татьяна Борисовна, к.м.н. [Tatyana B. Morgunova, MD, PhD], доцент кафедры эндокринологии №1 Института клинической медицины им. Н.В. Склифосовского ФГАОУ ВО «Первый МГМУ им. И.М. Сеченова» Минздрава России (Сеченовский Университет), Москва, Россия; адрес: 119991, Москва, ул. Трубецкая, д. 8, стр. 2 [address: 8-2 Trubetskaya str., Moscow 119991, Russia]; ORCID: http://orcid.org/0000-0003-1500-1586; eLibrary SPIN: 3705-8599; e-mail: tanmorgun@mail.ru
\end{abstract}

Зорина Анастасия Александровна [Anastasia A. Zorina], студентка, 5 курс, лечебный факультет ФГАОУ ВО «Первый МГМУ им. И.М. Сеченова» Минздрава России (Сеченовский Университет), Москва, Россия; ORCID: https://orcid.org/0000-0002-2234-5283; eLibrary SPIN: 5670-4957; e-mail: zorinavgustina@ya.ru

Малолеткина Екатерина Станиславовна [Ekaterina S. Maloletkina, MD], врач-эндокринолог эндокринологического терапевтического отделения №2, Университетская клиническая больница №2, ФГАОУ ВО «Первый МГМУ им. И.М. Сеченова» Минздрава России (Сеченовский Университет), Москва, Россия; ORCID: https://orcid.org/0000-0001-9881-5459; eLibrary SPIN: 6794-2220; e-mail: Maloletkinae@mail.ru Сыч Юлия Петровна, к.м.н. [Yulia P. Sytch, MD, PhD], ассистент кафедры эндокринологии №1 Института клинической медицины им. Н.В. Склифосовского ФГАОУ ВО «Первый МГМУ им. И.М. Сеченова» Минздрава России (Сеченовский Университет), Москва, Россия; ORCID: https://orcid.org/0000-0002-7000-0095; eLibrary SPIN: 3406-0978; e-mail: juliasytch@mail.ru 
Васильева Ариадна Васильевна [Ariadna V. Vasileva, MD], ассистент кафедры эндокринологии №1 Института клинической медицины им. Н.В. Склифосовского ФГАОУ ВО «Первый МГМУ им. И.М. Сеченова» Минздрава России (Сеченовский Университет), Москва, Россия; ORCID: https://orcid.org/0000-0001-7044-3921; eLibrary SPIN: 4270-8251; e-mail: avasileva.md@gmail.com

Фадеев Валентин Викторович, д.м.н., профессор, член-корр. PAH [Valentin V. Fadeyev, MD, PhD, Professor], заведующий кафедрой эндокринологии №1 Института клинической медицины им. Н.В. Склифосовского ФГАОУ ВО «Первый МГМУ им. И.М. Сеченова» Минздрава России (Сеченовский Университет), Москва, Россия; ORCID: http://orcid.org/0000-0002-3026-6315; eLibrary SPIN: 6825-8417; e-mail: walfad@mail.ru

\section{ЦИТИРОВАТЬ}

Моргунова Т.Б., Зорина А.А., Малолеткина Е.С., Сыч Ю.П., Васильева А.В., Фадеев В.В. Болезнь Грейвса у пациентки с ВИЧ-инфекцией, получающей антиретровирусную терапию // Проблемы эндокринологии. — 2020. - Т.66. - №3. C. 22-26. doi: https://doi.org/10.14341/probl12375

\section{FOR CITATION}

Morgunova TB, Zorina AA, Maloletkina ES, Sytch YP, Vasileva AV, Fadeyev VV. Graves' disease in HIV patient receiving antiretroviral therapy. Problems of Endocrinology. 2020;66(3):22-26. doi: https://doi.org/10.14341/probl12375 